\title{
A NOVEL SIGNAL PROCESSING METHOD BASED ON FREQUENCY MODALITY FOR INTRA-BODY MEDICAL INSTRUMENT TRACKING
}

\author{
Dimitrios A. Fotiadis ${ }^{1), 2)}$, Kostas Papathanasiou ${ }^{3)}$, Alexandros Astaras ${ }^{1)}$, \\ Panagiotis D. Bamidis ${ }^{1)}$, Anestis Kalfas ${ }^{4)}$ \\ 1) School of Medicine, Aristotle University of Thessaloniki, 54124 Thessaloniki, Greece, \\ \{dfotiadis, astaras, bamidis\}@med.auth.gr \\ 2) Department of Informatics Engineering, School of Applied Science, \\ Technological Education Institute of Western Macedonia, 52100 Kastoria, Greece, \\ d.fotiadis@kastoria.teikoz.gr, http://www.teiwm.gr/site/d.fotiadis \\ 3) Department of Physics, Aristotle University of Thessaloniki, 54124 Thessaloniki, Greece, \\ kostasp@physics.auth.gr, http://electronics.physics.auth.gr/tomeas/cv/papathanasiou_cv_en.pdf \\ 4) Department of Mechanical Engineering, Aristotle University of Thessaloniki, 54124 Thessaloniki, Greece, \\ akalfas@auth.gr, http://users.auth.gr/akalfas \\ 5) Department of Computer Science, American College of Thessaloniki, 55535 Pylea, Thessaloniki, Greece, \\ astaras@act.edu, https://angel.co/astaras
}

\begin{abstract}
Measuring the position of a medical instrument inside the human body can be performed with various methods. One option is to measure the phase shift of the signal originating from a transmitter embedded into the tip of the medical instrument, determining its displacement with respect to a set of stationary receivers. The phase shift is converted into a low frequency voltage with the use of a Phased Locked Loop (PLL). This voltage can subsequently be converted into displacement, providing the position of the medical instrument in one (1D), two (2D) and three (3D) dimensions using trilateration. The instrument's displacement can be defined in either the time or frequency domain. This paper presents a novel method for constant velocity displacement of the transmitter, using either the Locally Weighted Scatter-Plot Smoothing (LOWESS) curve fitting method or a Lomb-Scargle periodogram. The Lomb-Scargle periodogram is based on the least-squares power spectrum and can be used instead of waveform smoothing and measurement into the time domain, providing more precise and accurate measurement results as compared to LOWESS curve fitting method. Copyright (C) Research Institute for Intelligent Computer Systems, 2015. All rights reserved.
\end{abstract}

Keywords: Position tracking, medical instrument, PLL, LOWESS, Lomb-Scargle periodogram, error analysis.

\section{INTRODUCTION}

Position tracking is a technological field with a diverse variety of applications. Among those biomedical engineering applications related to medical surgery, such as cardiovascular, orthopedic and neurosurgical catheterization, the precise detection of tumors and biopsy needle insertion. In all aforementioned applications, knowing the precise position of a medical instrument's tip is often a requirement. The level of accuracy (minimum measurement error) and precision (minimum deviation across repeated measurements) varies among applications, however in most cases millimeter level accuracy and precision are more than adequate.

Optical and electromagnetic (EM) based tracking solutions nowadays dominate the field of biomedical engineering position tracking [1]. Each one of these has its advantages and disadvantages.
Optical tracking is a three dimensional (3D) localization technology, which is based on the monitoring of a defined distance measurement using two or more cameras. Each camera is equipped with a series of infrared (IR) LEDs which are periodically illuminating the measurement space with IR light. Medical instruments that need to be tracked are equipped with retro-reflective markers, which reflect the incoming IR light back to the cameras. Then, the IR reflections are detected by the cameras and are processed using geometrical methods in order to define the exact medical instrument's position. NDI Polaris family of optical measurement systems is one of the dominant on market today [2]. Optical tracking systems require clear line-of-sight (LOS) to exist between the medical instrument tracked and the control device. This prohibits the use of such a system in cases where an invasive medical instrument is to be tracked. 
Electromagnetic (EM) tracking systems are based on the use of a transmitter, located on the moving object being tracked, and a number of receivers located a short distance away (generally closer than $2 \mathrm{~m})$. The transmitted signal frequency is low, in the order of a few $\mathrm{kHz}$, so as to bypass multipath interference problems and attenuation of signal strength as it propagates through the human body.

EM systems generate a series of electromagnetic pulses from the field generator or "transmitter". The signals from the transmitter induce a voltage in the sensors that are attached to the medical instruments.

The transmitting and receiving antennae are a set of three coils perpendicular to each other, able to transmit and receive in three dimensions $(\mathrm{X}, \mathrm{Y}$ and $\mathrm{Z}$ ). Using triangulation, a method commonly used by GPS systems, the exact spatial position of a catheter's tip can be defined with subcentimeter accuracy.

Trackers for EM systems include small sensor coils that are attached to or are embedded into the medical instrument. Several technology generations of trackers are available commercially. These range from $1^{\text {st }}$ generation $\mathrm{AC}$ electromagnetic technology [3], later enhanced by $[4,5] ; 2^{\text {nd }}$ generation trackers introduced $3^{\text {rd }}$ generation DC magnetic technology [6].

EM based systems are able to operate without any requirement for LOS, but the presence of metallic objects in the near vicinity of the system [7] will interfere with measurement results and adversely affect system precision and accuracy.

Depending on the required level of precision, measurement of the position of a moving object may require the use of technological and mathematical error-analysis methods. An alternative and novel method, based on tracking the position of a medical instrument inside the human body has been presented in $[8,9]$. This methodology employed phase shift measurement of a high frequency electromagnetic transmission originating from the medical instrument that was then used to calculate its displacement with respect to reference receivers outside the human body. The receiver electronics was based on a phase-locked loop (PLL) implementation and the experimental measurements were performed using a plain, low frequency digital oscilloscope. The method's simplicity, relatively high precision, small size and low volume requirements, as well as a low manufacturing cost are considerable advantages compared to other solutions.

The system presented in $[8,9]$ was based on a method where the displacement of a transmitter was represented by a low frequency voltage signal output at the receiver end of the system. The oscilloscope used for the experimental evaluation of a descibed system $[8,9]$ allowed for exporting the voltage which represents transmitter displacement - into image format data files. These files were appropriately processed to provide voltage versus time waveforms and time series, further processed to yield precision and system accuracy metrics. These waveforms were smoothed with the use of the LOWESS method [10] and error analysis was performed in order to define the system's capability of performing accurate position tracking [9].

In this paper we present a novel data processing method which may be particularly applicable to biomedical engineering field data analysis, since measurements from safety critical operations are often opportunistic and unevenly sampled across the time domain [11]. Our experimental displacement data, which is extracted from the oscilloscope image format file, was an example of such an unevenly sampled data set. The power spectral density estimation of such a data set and more specifically the Lomb-Scargle periodogram analysis [12-14] provides quite interesting results, in a sense of better position tracking system accuracy and precision. In cases where the invasive medical instrument is displaced at constant velocity, which is a common operating scenario for surgical catheters, utilization of the Lomb-Scargle periodogram provides orders of magnitude of improvement in position tracking accuracy and precision. The experimental setup is described in Section 2. Next, the data processing method is analyzed in Section 3. In Section 4, the proposed frequency domain data processing method is explained. Finally, the conclusions are presented in Section 5.

\section{EXPERIMENTAL SETUP}

The experimental system setup is similar to the one described in [9]. A transmitter located on a wheeled platform is displaced at constant velocity with respect to a stationary receiver.

A transmitter embedded in a medical catheter's tip, being displaced with respect to a reference frame comprising several stationary receivers, will generate pure sinusoid signals at the receivers output if moving at constant velocity. Typical Intravascular Ultrasound (IVUS) catheters for example are moving inside the human body with the assistance of a motor driven guide wire and a pull-back velocity of the catheter in the order of 0.5 or $1 \mathrm{~mm}$ per second [15].

The transmitter's displacement is represented by a Doppler X-Band receiver intermediate frequency (IF) signal. This signal is captured with the use of a low frequency digital oscilloscope. The captured signal is then exported to a PC with the use of a USB interface and an appropriate software application. 


\section{DATA PROCESSING}

The file transferred from the oscilloscope to the interconnected PC is a raster format (.BMP) screen capture. This kind of data file format does not provide the capability to process the experimental data, thus a data conversion method was used, in order to finally obtain an appropriate file format with measurable elements.

This image data file was subsequently converted into a vector format image and an $x-y$ data set was extracted in the form of a comma separated value (.CSV) file format. The $\mathrm{x}$ and $\mathrm{y}$ axis values represent time and voltage respectively. Such a file provides the data for amplitude and time normalized graph, representing the displacement voltage over a specific time period. The major weakness of this conversion procedure is that the data series waveform is unevenly sampled.

The displacement equivalent voltage graph is a noisy signal, from which it is difficult to obtain the necessary information in order to evaluate the system's overall accuracy and precision. A curve fitting or regression analysis method was used in order to filter noise from the signal and provide the capability for a statistical evaluation of our system. These methods are typically used to define the optimum curve which fits an experimental data series. In most cases the curve fitting result is a series of equations used to specify various points along a curve. In other cases however, this method does not provide the aforementioned equation definition, but rather a curve which smooth's data in a manner which resembles hardware filtering.

Existing curve fitting methods can be categorized in three primary categories: least squares curve fits, nonlinear curve fits and smoothing curve fits. The first two methods provide an equation which describes the resulting curve. The third method, by contrast does not generate an equation since it is impossible to always have a single equation describing the resulting curve. The smoothing method is used in order to have a much more uniform curve, actually filtering noise out from the initial data waveform.

The method used was LOWESS (Locally Weighted Scatter-Plot Smoothing), a method similar to the least squared error smoothing method. LOWESS is a non-parametric regression method. This method is based on data analysis aiming at the creation of a set of points along a curve. This curve is described in the form of Cartesian X-Y coordinates, the $X$ variable representing time. Since the experimental data waveforms captured from the oscilloscope have time represented along the $\mathrm{X}$ axis this method constitutes an improvement with respect to the least squares smoothing method.
In LOWESS method locally weighted linear regression is used in order to smooth measured signal waveforms [10]. Initially the regression weights are computed, for each sampled data point. The weights $w$ for each data point are given by:

$$
w_{i}=\left(1-\left(\frac{x-x_{i}}{d(x)}\right)^{3}\right)^{3}
$$

where $x$ is the predictor value associated with the response value to be smoothed, $x_{i}$ are the neighbors that are nearest at $x$ as defined by the span and $d(x)$ is the distance along the abscissa from $x$ to the most distant predictor value within the span.

Next, a weighted linear least squares regression is performed into the data set. The regression employs a first degree polynomial.

Finally, the smoothed value is given by the weighted regression at the predictor value of interest.

The LOWESS method is superior for unevenly sampled data, whereas the least squares smoothing method is not.

An important parameter concerning the LOWESS method is the smoothing factor $\alpha$. This factor is a number between $(v+1) / n$ and 1 , with $v$ denotes the degree of the local polynomial for $n$ number of data points. The value of $a$ is the proportion of data used in each fit. A value of $a$ in the order of 0.25 was used for smoothing the measured displacement data series.

A Zero Intermediate Frequency (ZIF) signal [9] measured with a low frequency digital oscilloscope, at $4 \mathrm{~cm} / \mathrm{s}$ constant transmitter velocity, together with a smoothed ZIF signal is depicted in Fig. 1.

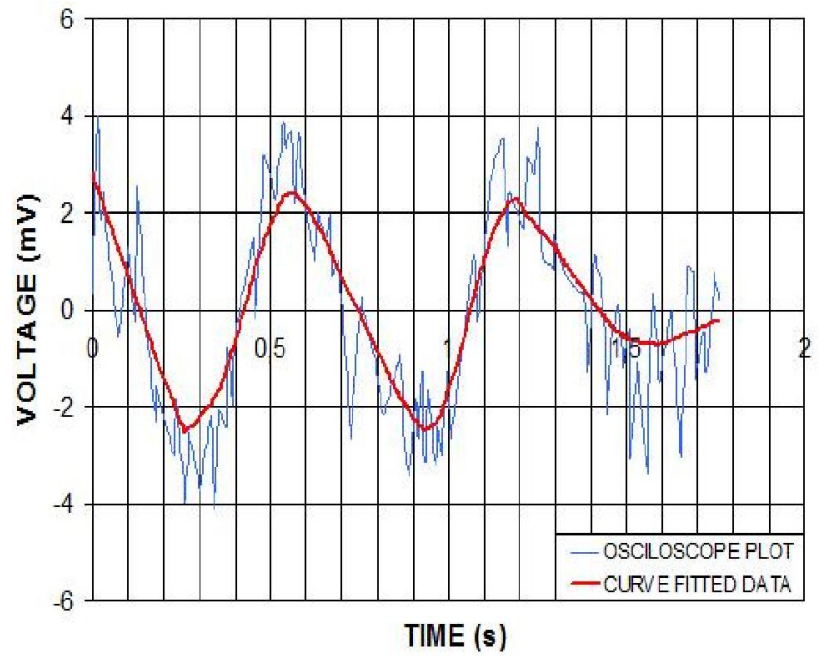

Fig. 1 - ZIF oscilloscope captured signal in comparison to smoothed with LOWESS algorithm signal for $4 \mathrm{~cm} / \mathrm{s}$ constant transmitter velocity. 
After smoothing the signal, applicable accuracy and precision metrics allowed us to qualitatively evaluate our system by performing calculations into the time domain.

The Standard Deviation $(S D)$ of measurements or $\sigma=0.653 \mathrm{~mm}$ and the typical error is $\frac{\sigma}{\sqrt{n}}=0.206 \mathrm{~mm}$, where $n$ is the number of measured waveforms that were smoothed.

Concerning system precision, in $99.7 \%$ of measurements, measurement error will be less or equal to $\pm 3 \sigma= \pm 1.959 \mathrm{~mm}$. The $95 \%$ confidence interval bound is calculated as $\pm 0.4 \mathrm{~mm}$ [16].

\section{FREQUENCY DOMAIN DATA PROCESSING}

Keeping in mind that experimental data from a medical instrument moving inside the human body is most likely noisy and affected by various sources of interference, we seek to utilise efficient error analysis algorithms, in order to extract as much useful information from the data as possible [17]. Spectral analysis or frequency domain processing is a valuable digital signal processing tool, which was used for our experimental data.

As mentioned earlier, the signal provided from the image data file conversion is unevenly sampled. Interpolation to resample the signal uniformly is commonly used and reported in scientific literature [18]. Spectrum analysis methods such as Fast Fourier Transform (FFT) are subsequently performed, in order to acquire the spectrum content of the signal and obtain further valuable information from the frequency domain.

Interpolation of the system ZIF signal was initially performed. Afterwards, Fast Fourier Transform (FFT) was applied to the evenly sampled signal. An initial estimation of the primary frequency of the signal in the order of $1.5 \mathrm{~Hz}$ was obtained from the time domain signal, thus all higher frequencies were securely filtered out. Then Inverse Fast Fourier Transform (IFFT) is performed, in order to acquire a new, noise-filtered reconstruction of the signal. FFT, signal filtering and IFFT were performed using the MATLAB signal processing toolbox.

As expected, the reconstructed, noise-filtered signal associated with a constant transmitter velocity of $4 \mathrm{~cm} / \mathrm{s}-$ as depicted in Fig. 2 - is a close approximation of a sinusoidal curve.

This is the expected initial ZIF graph, since the system transmitter is displaced with constant velocity away from the receiver. Basic PLL theory and previous conducted system simulation proves the fact that the system's output voltage is a sinusoidal signal, for constant transmitter velocity $[9,19,20]$. Unfortunately the final reconstructed signal is not as close an approximation to a sinusoidal signal as hoped in order for it to be used for qualitative system characterization.

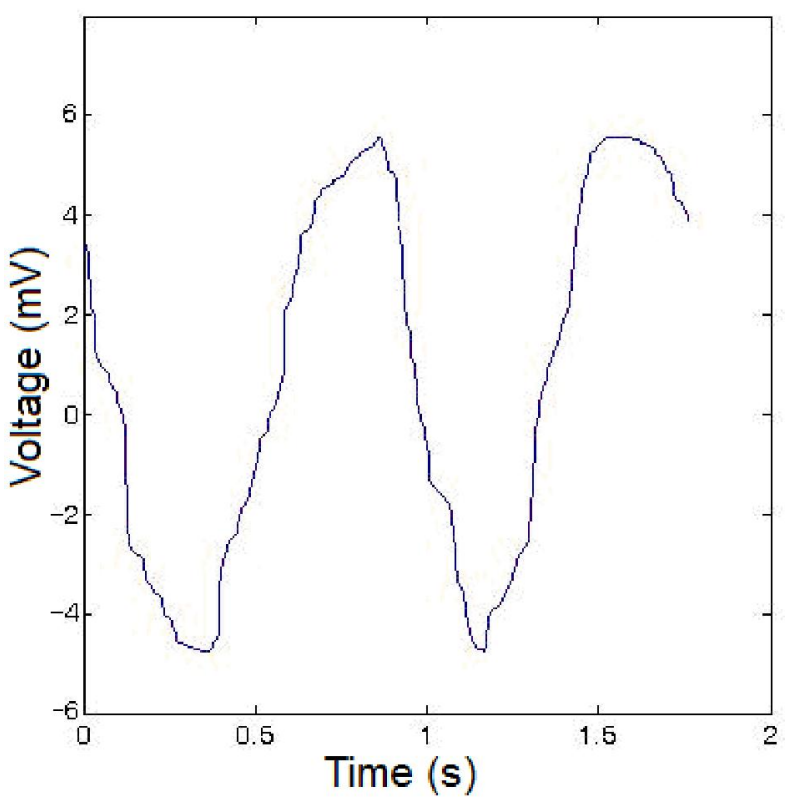

Fig. 2 - ZIF signal for $4 \mathrm{~cm} / \mathrm{s}$ constant transmitter velocity after FFT - low pass filtering - IFFT Matlab processing.

Since traditional spectral estimators need even sampling rates, unevenly spaced series need to be interpolated and resampled before spectral analysis. By contrast, the most common signal spectrum analysis method for small amplitude signals with periodicity such as is the measurement data for our case is the Lomb-Scargle periodogram (often called LSSA: Least-Squares Spectral Analysis) [12-14]. It is a method which does not require interpolation and resampling [21]. A periodogram is actually a graph showing frequency power for a specific spectrum of frequencies. For time-series of $M$ data points, $Y_{i}=Y\left(t_{i}\right)$ sampled at time instances $t_{i}$, where $i=1,2, \ldots, M$, with a mean value of $\bar{Y}$, the LombScargle periodogram is computed from Eq. (2):

$$
\begin{aligned}
& P N(\omega)=\frac{1}{2 \sigma^{2}}\left\{\frac{\left[\sum_{i}\left(Y_{i}-\bar{Y}\right) \cos \omega\left(t_{i}-\tau\right)\right]^{2}}{\sum_{i} \cos ^{2} \omega\left(t_{i}-\tau\right)}\right. \\
& \left.+\frac{\left[\sum_{i}\left(Y_{i}-\bar{Y}\right) \sin \omega\left(t_{i}-\tau\right)\right]^{2}}{\sum_{i} \sin ^{2} \omega\left(t_{i}-\tau\right)}\right\}
\end{aligned}
$$

where $\tau$ is given by Eq. (3):

$$
\tau=(1 / 2 \omega) \tan ^{-1}\left\lfloor\Sigma_{i} \sin 2 \omega t_{i} / \Sigma_{i} \cos 2 \omega t_{i}\right\rfloor
$$


$P N$ is the normalized power as function of angular frequency $(\omega=2 \pi / P)$ for all the periods $(P)$ which were totally tested. When the term "normalized" is mentioned, it actually refers to $\sigma^{2}$, which is the total variance of the data $Y\left(t_{i}\right)$ and permits the determination of the statistical significance of peaks in a periodogram [13]. On the other hand, the term $t$ is used in order to adjust for phase shifts caused by unevenly sampled data and was first introduced by Lomb [12]. A peak in such a periodogram indicates a frequency with significant periodicity. Of course, for evenly sampled data sets, the results of Lomb-Scargle periodogram are exactly the same as for the classical power spectrum [22].

When the data are unevenly sampled, as is our experimental case, there are specific differences between the Lomb-Scargle periodogram and FFT analysis, such as: a) the Lomb-Scargle periodogram method weights data points, while the FFT method weights frequency intervals, b) the Lomb Scargle periodogram can be used with unevenly sampled data, while the FFT cannot c) using the Lomb Scargle periodogram method we have no data imputation, which is not the case with FFT and d) any number of data points can be used in a Lomb Scargle periodogram, while the data points in FFT is $2^{\mathrm{N}}$ and any missing points are zero- padded.

Utilisation of the Lomb Scargle periodogram for the same ZIF signal that was used as input for time and frequency domain analysis that was shown above provided the Lomb Scargle periodogram shown in Fig. 3.

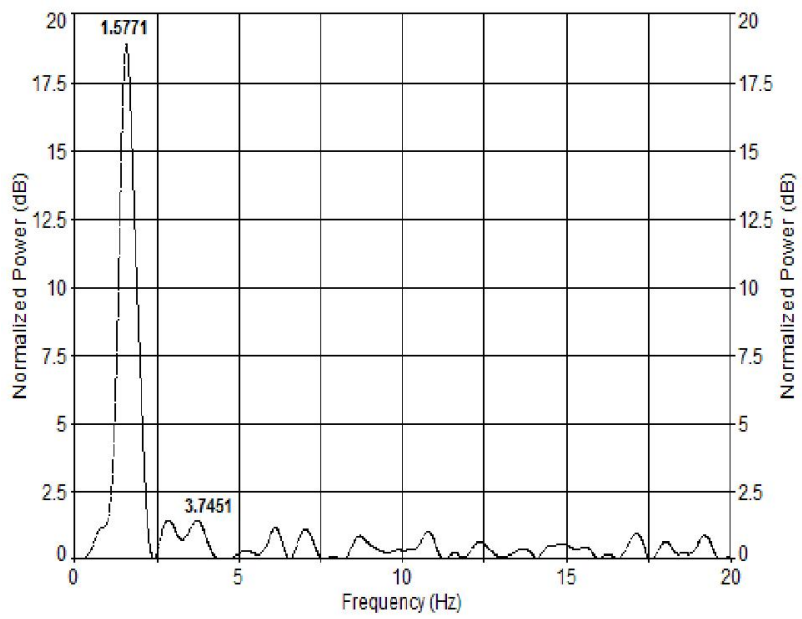

Fig. 3 - Lomb-Scargle periodogram of ZIF signal, for $4 \mathrm{~cm} / \mathrm{s}$ constant transmitter velocity.

It can be clearly sheen that the primary signal frequency has multiple orders of higher amplitude in comparison with all other harmonics. Using this frequency one can easily assume that the ZIF signal is a sinusoid with frequency $1.5771 \mathrm{~Hz}$. This signal representation together with an unfiltered, noisy ZIF signal is shown in Fig. 4.

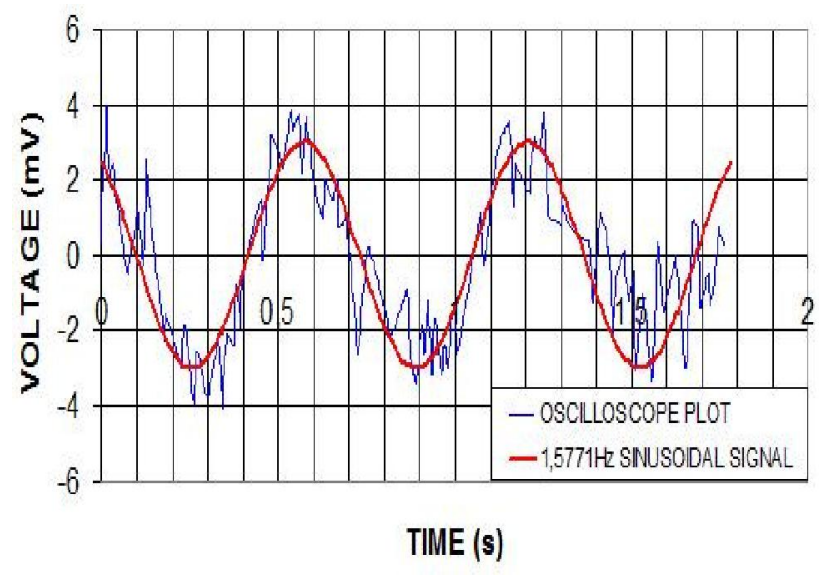

Fig. 4 - ZIF signal captured with a digital oscilloscope together with a $1.5771 \mathrm{~Hz}$ frequency sinusoidal signal representation, for $4 \mathrm{~cm} / \mathrm{s}$ constant transmitter velocity.

Performing the same frequency extraction for the entire set of 100 measurements, the subsequent calculation of the standard deviation, typical error, $95 \%$ confidence interval and other statistical metrics proves the system's robustness and increased precision. The calculated precision is subsequently compared to the data provided by time domain analysis using the LOWESS smoothing method. In the Lomb Scargle periodogram method case, the Standard Deviation $(S D)$ or $\sigma=0.2 \mathrm{~mm}$ and the typical error $\frac{\sigma}{\sqrt{n}}=0.063 \mathrm{~mm}$, where $n$ is the number of measured waveforms that were processed using Lomb Scargle periodogram. Concerning system precision, in $99.7 \%$ of measurements, measurement error will be less or equal to $\pm 3 \sigma= \pm 0.6 \mathrm{~mm}$. The $95 \%$ confidence interval bound is calculated to be $\pm 0.123 \mathrm{~mm}$.

Table 1 shows the precision statistical results from both methods (LOWESS and Lomb Scargle periodogram).

Table 1. Processing methods comparison.

\begin{tabular}{|c|c|c|c|}
\hline $\begin{array}{c}\text { Processing } \\
\text { method }\end{array}$ & $\boldsymbol{\sigma}(\mathbf{m m})$ & $\frac{\boldsymbol{\sigma}}{\sqrt{\mathbf{n}}(\mathbf{m m})}$ & $\begin{array}{c}\mathbf{9 5 \%} \\
\mathbf{( m m})\end{array}$ \\
\hline LOWESS & 0.653 & 0.206 & \pm 0.4 \\
\hline $\begin{array}{c}\text { Lomb Scargle } \\
\text { periodogram }\end{array}$ & 0.2 & 0.063 & \pm 0.123 \\
\hline
\end{tabular}

Since our system can only be compared with electromagnetic medical tracking systems, due to the fact that they are the sole systems that can operate 
without the transmitter having clear line-of-sight (LOS) with the associated receivers, a comparison between our implementation and a typical electromagnetic medical position tracking system can be performed, as far as standard deviation, or 95\% CI concerns. A study performed at Oulu University Hospital presenting electromagnetic systems characteristics was presented into [23], measuring position tracking precision and accuracy, in a similar way that was performed for our system. The precision of electromagnetic tracking systems that were evaluated shows a $0.13 \mathrm{~mm}$ standard deviation which is comparable with our proposed system's standard deviation when Lomb Scargle periodogram method was employed, while the $95 \%$ CI of the electromagnetic systems was $0.76 \mathrm{~mm}$, while our system's 95\% CI was measured $0.123 \mathrm{~mm}$.

\section{CONCLUSIONS}

Both LOWESS and Lomb Scargle periodogram methods can be used in order to obtain position tracking precise results. It is preferable to employ the Lomb Scargle periodogram method in cases where the transmitter or the medical instrument is being displaced with constant velocity. LOWESS is the preferred method in cases where transmitter velocity is not constant.

In cases such as a medical catheter, the displacement velocity is guarantied to be constant and the Lomb Scargle periodogram method can provide optimum precision to the position tracking system.

In cases where the medical instrument is displaced manually by a surgeon, without motor pullback assistance, the LOWESS method appears to be an optimum solution for filtering noise out of the displacement measurement. Still, the Lomb Scargle periodogram method can also be efficiently used in cases where the physician manually displaces the catheter at a relatively constant velocity. The system's precision in the latter scenario is yet to be experimentally investigated.

\section{REFERENCES}

[1] T. M. Peters and K. R. Cleary, Image-guided Interventions: Technology and Applications. Springer, 2008.

[2] Medical Polaris Optical Tracking Systems, [Online], Available: http://www.ndigital.com/ medical/products/polaris-family/, [Accessed: 04-Apr-2015].

[3] Polhemus Electromagnetics, [Online]. Available: http://polhemus.com/applications/ electromagnetics/, [Accessed: 04-Apr-2015].
[4] 'superDimension ${ }^{\mathrm{TM}}$ Navigation System | Medtronic PLC Medtronic PLC', [Online], Available: http://superdimension.com/products/ superdimension-system/. [Accessed: 04-Apr2015].

[5] Medical Aurora, [Online], Available: http://www.ndigital.com/medical/products/auro ra/, [Accessed: 04-Apr-2015].

[6] Ascension Technology Corporation, [Online], Available: http://www.ascension-tech.com/, [Accessed: 04-Apr-2015].

[7] F. Stevens, M. A. Conditt, N. Kulkarni, S. K. Ismaily, P. C. Noble, and D. R. Lionberger, Minimizing electromagnetic interference from surgical instruments on electromagnetic surgical navigation, Clin. Orthop., (468) 8 (Aug. 2010), pp. 2244-2250.

[8] D. A. Fotiadis, A. Astaras, A. Kalfas, K. Papathanasiou, and P. Bamidis, Displacement measurement of a medical instrument inside the human body, in Proceedings of the $13^{\text {th }}$ Mediterranean Conference on Medical and Biological Engineering and Computing MEDICON'2013, Sevilla, Spain, 2013.

[9] D. Fotiadis, A. Astaras, P. D. Bamidis, K. Papathanasiou, and A. Kalfas, Experimental evaluation of an invasive medical instrument based on a displacement measurement system, IEEE J. Biomed. Health Inform., (PP) 99 (2014), pp. 1-1.

[10] W. S. Cleveland, Robust locally weighted regression and smoothing scatterplots, J. Am. Stat. Assoc., (74) 368 (1979), pp. 829-836.

[11] D. S. Fonseca, A. D. Netto, R. B. Ferreira, and A. M. F. L. M. de Sa, Lomb-scargle periodogram applied to heart rate variability study, in Biosignals and Biorobotics Conference (BRC), 2013 ISSNIP, 2013, pp. 1-4.

[12] N. R. Lomb, Least-squares frequency analysis of unequally spaced data, Astrophys. Space Sci., (39) 2 (Feb. 1976), pp. 447-462.

[13] J. D. Scargle, Studies in astronomical time series analysis. II - Statistical aspects of spectral analysis of unevenly spaced data, Astrophys. J., (263) (Dec. 1982), p. 835.

[14] P. Vaníček, Further development and properties of the spectral analysis by least-squares, Astrophys. Space Sci., (12) 1 (Jul. 1971), pp. 10-33. 
[15] S. Watson and K. Gorski, Invasive Cardiology: A Manual for Cath Lab Personnel: A Manual for Cath Lab Personnel, Jones \& Bartlett Learning, 2010.

[16] J. R. Taylor, An Introduction to Error Analysis: The Study of Uncertainties in Physical Measurements, 2nd ed., University Science Books, 1996.

[17] S. Kazemi, A. Ghorbani, H. Amindavar, and C. Li, Cyclostationary approach to Doppler radar heart and respiration rates monitoring with body motion cancelation using Radar Doppler System, Biomed. Signal Process. Control, (13) (Sep. 2014), pp. 79-88.

[18] M. L. Stavrinou, G. C. Sakellaropoulos, E. Trachani, V. Sirrou, P. Polychronopoulos, G. Nikiforidis, and E. Chroni, Methodological issues in the spectral analysis of the heart rate variability: Application in patients with epilepsy, Biomed. Signal Process. Control, (13) (Sep. 2014), pp. 1-7.

[19] D. Banerjee, PLL Performance, Simulation and Design, Fourth Edition, 4th ed., Dog Ear Publishing, LLC, 2006.

[20] F. M. Gardner, Phaselock Techniques, 3rd ed., Wiley-Interscience, 2005.

[21] P. Laguna, G. B. Moody, and R. G. Mark, Power spectral density of unevenly sampled data by least-square analysis: performance and application to heart rate signals, IEEE Trans. Biomed. Eng., (45) 6 (Jun. 1998), pp. 698-715.

[22] H. P. Van Dongen, E. Olofsen, J. H. VanHartevelt, and E. W. Kruyt, Searching for biological rhythms: peak detection in the periodogram of unequally spaced data, J. Biol. Rhythms, (14) 6 (Dec. 1999), pp. 617-620.

[23] T. Koivukangas, J. P. Katisko, and J. P. Koivukangas, Technical accuracy of optical and the electromagnetic tracking systems, SpringerPlus, (2) 1, p. 90, Mar. 2013.

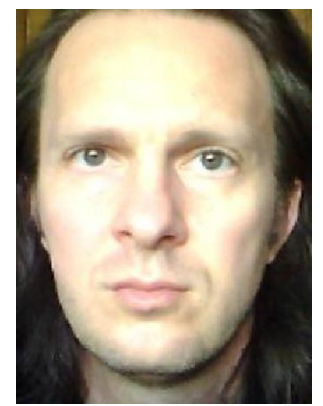

Dimitris A. Fotiadis (b. 1973) received his B.SC. in electronics engineering from Technological Education Institute of Thessaloniki Greece in 1996 and his M.Sc. in Electronics (Digital Systems) from University of Hertfordshire, U.K., in 1997. Currently he is Lecturer at the Department of Informatics
Engineering, Kastoria Branch, Technological Education Institute of Western Macedonia (TEI WM), Greece and a PhD candidate at the Medical Informatics Lab, School of Medicine, Aristotle University of Thessaloniki, Greece. His research interests are mainly concentrated on RF positioning and usage into biomedical field and implantable $R F$ systems, biomedical systems instrumentation, microcontrollers/ microprocessors biomedical robotics design and implementation.

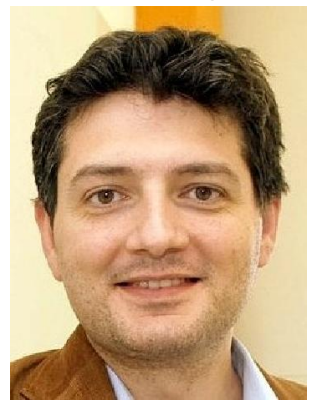

Alexandros Astaras (b. 1971) received his B.A. degree in Physics from Oberlin College, Oberlin, OH USA in 1995 and his $P h D$ degree from the University of Edinburgh, Edinburgh, U.K. in 2004. Currently he is a Research Associate in Biomedical Engineering at the Medical Physics Lab, School of Medicine, Aristotle University of Thessaloniki, Greece, as well as a part-time lecturer in Electronics at the Department of Automation, Technological Educational Institute of Thessaloniki, Greece and an Adjunct Professor in Computer Science at the American College of Thessaloniki, Greece. His research interests revolve around biomedical diagnostics, medical instrumentation, and mixed signal system-on-chip VLSI design.

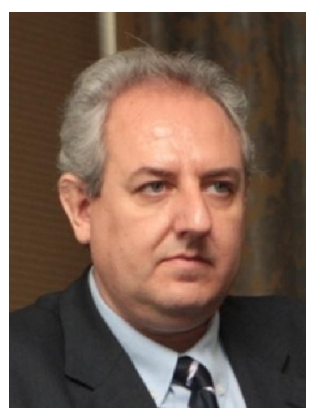

Panagiotis D. Bamidis (b. 1968) received the Diploma degree in physics from the Aristotle University of Thessaloniki (AUTH), Thessaloniki, Greece in 1990, the M.Sc. (with distinction) degree in medical physics from the University of Surrey, Guildford, U.K., in 1992, and the Ph.D. degree in bioelectromagnetism and functional brain analysis and imaging from the Open University, Milton Keynes, U.K., in 1996.

$\mathrm{He}$ is currently an Assist. Professor in Medical Education Informatics within the Laboratory of Medical Physics, Medical School, AUTH. His research interests are within assistive technologies (silverscience, silvergaming, mobile health, decision support, avatars), technology enhanced learning in Medical Education (web2.0, semantic web, serious games, virtual patients, PBL, learning analytics) and Affective and Physiological Computing and $\mathrm{HCl}$, (bio)medical informatics with emphasis on neurophysiological sensing and health information management (open health big data), and Affective Neuroscience. 


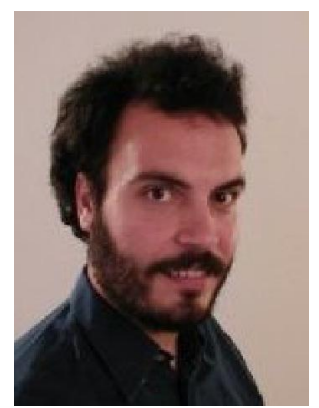

Kostas Papathanasiou (b. 1970) received his Degree in Electrical Engineering in 1992 and his M.Sc. in Electrical \& Electronic Engineering in 1994 from University of Patras, Greece, while he received his $\mathrm{PhD}$ from University of Edinburgh, U.K. in 1998. Currently he is a Lecturer at the Electronics and Computers Lab, Physics Department, Aristotle University of Thessaloniki, Greece. His research interests include analog and RF integrated systems, implantable low-power devices, biomedical signal monitoring, analog uses of floating gates, CAD techniques for parasitic extraction, analog design automation and porting, device modeling and mismatch.

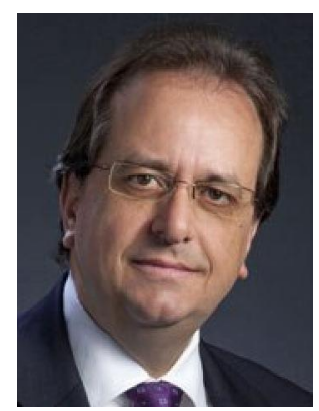

Anestis Kalfas (b. 1968) is an Assistant Professor in Turbomachinery. He belongs to the teaching staff of the Laboratory of Fluid Mechanics and Turbo- machinery of the Department of Mechanical Engineering at the Aristotle University of Thessaloniki.

Anestis Kalfas received his
PhD in Turbomachinery Aero-dynamics from Cranfield University in 1994 and his Dipl.-Ing. Mech. Eng. Aristotle University of his native Thessaloniki. He worked as a Research Associate at the Whittle Lab., University of Cambridge and as an Aircraft Engineer at the Hellenic Air Force. He has been a Senior Scientist at the Turbomachinery Laboratory of the Swiss Federal Institute of Technology in Zurich, since July 2000 where he lectured in Turbomachinery Design.

Dr Kalfas is active in the areas of axial steam and gas-turbine aerodynamics, gas-turbine performance and power plant optimization, boundary layer transition and turbulence and novel aerodynamic probe technology. 